\title{
Cubic Interactions of Massless Bosonic Fields in Three Dimensions
}

\author{
Karapet Mkrtchyan* \\ Max-Planck-Institut für Gravitationsphysik (Albert-Einstein-Institut), Am Mühlenberg 1, 14476 Golm, Germany
}

(Received 9 January 2018; revised manuscript received 30 March 2018; published 29 May 2018)

\begin{abstract}
In this Letter, we take the first step towards construction of nontrivial Lagrangian theories of higher-spin gravity in a metriclike formulation in three dimensions. The crucial feature of a metriclike formulation is that it is known how to incorporate matter interactions into the description. We derive a complete classification of cubic interactions for arbitrary triples $s_{1}, s_{2}, s_{3}$ of massless fields, which are the building blocks of any interacting theory with massless higher spins. We find that there is, at most, one vertex for any given triple of spins in 3D (with one exception, $s_{1}=s_{2}=s_{3}=1$, which allows for two vertices). Remarkably, there are no vertices for spin values that do not respect strict triangle inequalities and contain at least two spins greater than one. This translates into selection rules for three-point functions of higherspin conserved currents in two dimensional conformal field theory. Furthermore, universal coupling to gravity for any spin is derived. Last, we argue that this classification persists in arbitrary Einstein backgrounds.
\end{abstract}

DOI: $10.1103 /$ PhysRevLett.120.221601

In the quest for a simple model of quantum gravity, particular optimism is related to higher-spin (HS) gravity theories in three dimensions (see, e.g., [1,2]). This is related to the fact that these theories bypass all the no-go theorems that put stringent constraints on HS gravity theories in $D \geq 4$. Not only do they allow for Lagrangian formulation [3], finite HS spectrum [4], Minkowski background [5], and color decoration [6], which are problematic for known theories in higher dimensions, but they also allow for nontrivial solutions [7] and possess infinite-dimensional asymptotic symmetries $[4,8]$ familiar from two dimensional conformal field theories (CFTs). Despite all of these simplifying properties, one important but basic problem still remains to be understood in these theories. It is the compatibility of matter coupling with the local Lagrangian formulation. In this Letter, we make the first step towards the answer to this question.

Even though matter-coupled HS gravity [2] has been known for twenty years and is at the center of the conjectured [9] duality between HS gravity on $\mathrm{AdS}_{3}$ and $\mathrm{CFT}_{2}$ models with $W$ symmetries, a Lagrangian description is still missing. The simplicity of general relativity and (colored) HS Gravity in three dimensions [2] formulated as Chern-Simons theories with noncompact gauge groups [10] is lost as soon as one adds matter into the picture. A simpler set up for addressing matter coupling is

Published by the American Physical Society under the terms of the Creative Commons Attribution 4.0 International license. Further distribution of this work must maintain attribution to the author(s) and the published article's title, journal citation, and DOI. Funded by SCOAP . metriclike formulation $[1,11,12]$, where, no longer making use of Chern-Simons actions, one loses the simplicity of the gauge sector.

The question of the Lagrangian formulation of HS theories is a long standing puzzle, addressed, in particular, through attempts for perturbative constructions of the action, in the spirit of the so-called Fronsdal program [13], that resulted in full classification [14,15] of cubic interactions in dimensions greater than three (see, also, [16-18] for generating functions and discussion about possible relation to string theory). This classification is in one-to-one correspondence with that of conformal threepoint correlators of conserved currents in dimensions lower by one via the AdS/CFT dictionary $[15,19]$.

In three dimensions, unlike in higher dimensions, HS interacting theories do not require a nonzero cosmological constant $[20,21]$. Together with the fact that the Fronsdal program is technically simpler in Minkowski space compared to (A)dS, this makes the three dimensional flat space a preferred playground for the problem of Lagrangian formulation for nonlinear HS theories with matter content. Still, the systematics of three-linear interactions in $D=3$ is not yet known. Indeed, there are only a handful of works on HS interactions in three dimensions in the metriclike formulation (see, e.g., [1,11,12]). In this Letter, we start an investigation in this direction, proposing a classification of parity-even cubic vertices.

The main technical difference between the threedimensional and the higher-dimensional classifications of cubic vertices for HS fields is that in $D=3$ there exist Schouten identities, that should be taken into account in the covariant Noether equations for cubic vertices. Relevant Scouthen identities exist for cubic vertices of symmetric 
tensor fields in $D \leq 4$. Taking them into account has been shown to result in less vertices in $D=4$ compared to those in $D>4$ [15]. As we will show in this work, the consequences in $D=3$ are even more drastic.

The cubic vertices for massless fields can be constructed order by order in traces and divergences of the fields involved [14], starting from the piece that does not involve any trace or divergence, which is usually referred to as the traceless-transverse (TT) vertex. In this Letter, we will work at the level of TT fields. Therefore, we do not solve the problem of finding off-shell vertices, but rather that of classifying them. In a sense, the results of this Letter can be regarded as the three-dimensional analogue of the lightcone classification in higher dimensions [22,23].

Covariant classification [14] of cubic vertices in dimensions greater than three has been reviewed using simplified notation in [15]. We will adopt the same notations here. A spin $s$ massless field is parametrized by a symmetric $s$ th rank tensor $\phi_{\mu_{1}, \ldots, \mu_{s}}$. We will contract all the indices of these fields with vector variables, $a^{\mu}$

$$
\phi^{(s)}(x, a)=\frac{1}{s !} \phi_{\mu_{1}, \ldots, \mu_{s}} a^{\mu_{1}} \cdots a^{\mu_{s}},
$$

to make the symmetry of indices manifest, as well as to simplify index contractions between the fields. We follow the Noether procedure, assuming that the Lagrangian can be expanded in powers of a small parameter $g$, starting from the free action [13], given by $\mathcal{L}^{(2)}$

$$
\mathcal{L}=\mathcal{L}^{(2)}+g \mathcal{L}^{(3)}+O\left(g^{2}\right) .
$$

The cubic action is a sum of different vertices

$$
\mathcal{L}^{(3)}=\sum_{\left\{s_{i}\right\}, n} g_{s_{1}, s_{2}, s_{3}}^{n} \mathcal{L}_{s_{1}, s_{2}, s_{3}}^{n},
$$

where $n$ is a parameter that counts independent vertices for a given triple of spins $s_{1} \geq s_{2} \geq s_{3}$ (number of free parameters, that are not fixed by the requirement of gauge invariance). In the following, we will completely discard the terms proportional to traces, divergences, Laplacian operators, and total derivatives and use the equals sign "=" between two vertex operators that are equivalent modulo terms containing these operators. In this way, we will only keep track of TT terms. The building blocks of TT cubic vertices are the following operators of scalar contractions between the fields $\phi_{i}\left(x_{i}, a_{i}\right)(i=1,2,3)$, and derivatives acting on them (see [15])

$$
y_{i}=\partial_{a_{i}} \cdot \partial_{x_{i+1}}, \quad z_{i}=\partial_{a_{i+1}} \cdot \partial_{a_{i-1}}, \quad(i+3 \equiv i) .
$$

Note that this choice of variables fixes the partial integration freedom and the field redefinition freedom, as in $[14,15]$. The operator of gauge variation, $\delta \phi_{i}=a_{i} \cdot \partial_{i} \epsilon_{i}$, acting on the TT vertex gives

$$
\delta_{i} \mathcal{L}^{(3)} \equiv\left(y_{i-1} \partial_{z_{i+1}}-y_{i+1} \partial_{z_{i-1}}\right) \mathcal{L}^{(3)}=0,
$$

which should vanish for on-shell TT fields. The solution to (5) in any dimensions is given by

$\mathcal{L}^{(3)}=\mathcal{V}\left(y_{i}, G\right) \phi_{1} \phi_{2} \phi_{3}, \quad G=y_{1} z_{1}+y_{2} z_{2}+y_{3} z_{3}$.

For given spins $s_{1} \geq s_{2} \geq s_{3}$, we have $\left(n=0,1, \ldots, s_{3}\right)$

$$
\mathcal{V}_{s_{1}, s_{2}, s_{3}}^{n}=g_{s_{1}, s_{2}, s_{3}}^{n} y_{1}^{s_{1}-n} y_{2}^{s_{2}-n} y_{3}^{s_{3}-n} G^{n} .
$$

Schouten identities and $3 D$ vertices.-The Schouten identities in three dimensions are contractions of arbitrary tensors with generalized Kronecker delta

$$
\delta_{\nu_{1}, \ldots, \nu_{4}}^{\mu_{1}, \ldots, \mu_{4}} \equiv 4 ! \delta_{\left[\mu_{1}\right.}^{\left[\nu_{1}\right.} \delta_{\mu_{2}}^{\nu_{2}} \delta_{\mu_{3}}^{\nu_{3}} \delta_{\left.\mu_{4}\right]}^{\left.\nu_{4}\right]} \equiv 0,
$$

where square brackets denote complete antisymmetrization. Such identities allow for the existence of additional gauge invariant vertices as compared to (7), namely, those which obey

$$
\delta_{i} \mathcal{V}=\text { Scouten identities } \equiv 0,
$$

for all $i$. One can systematically construct all the elementary Schouten identities as all possible contractions of (8) with operators $\partial_{a_{i}^{\mu}}, \partial_{\nu}^{i}(D=3)$. The complete list of parity-even elementary Schouten identities is given as

$$
\begin{gathered}
y_{i} z_{i} G-y_{i-1} z_{i-1} y_{i+1} z_{i+1}=0, \quad\left(G-y_{i} z_{i}\right)^{2}=0, \\
y_{i} y_{i \pm 1}\left(G-y_{i} z_{i}\right)=0, \\
y_{i}^{2} y_{i+1}^{2}=0, \quad y_{i}^{2} y_{i+1} y_{i-1}=0 .
\end{gathered}
$$

Note that we have grouped the identities into two-, three-, and four-derivative expressions.

In the following, we will state the results on classification of parity-even cubic vertices of massless fields in three dimensions. More details on their derivation are provided in [24].

Vertices with scalar and Maxwell fields.-To start with, we take the simplest example, where one of the fields is a scalar $\left(s_{1} \geq s_{2} \geq s_{3}=0\right)$. Our analysis suggests, that there are only two Lorentz invariant vertex operators compatible with gauge symmetry of massless fields with spin. The first one is

$$
\mathcal{V}_{s, 0,0}=y_{1}^{s},
$$

while the second one is

$$
\mathcal{V}_{s, 1,0}=y_{1}^{s} y_{2}
$$

We find that for the vertices involving scalar fields, the difference in three dimensions as compared to higher 
dimensions is the absence of vertices of interactions of the scalar with two fields of spins both greater than one.

Now, we turn to the next simple example-vertices with Maxwell fields $\left(s_{1} \geq s_{2} \geq s_{3}=1\right)$. We find two nontrivial solutions in this case. First, one requires $s_{2}=1$, and is the same as in higher dimensions

$$
\mathcal{V}_{s, 1,1}=y_{1}^{s-1} G,
$$

and reproduces the Yang-Mills vertex for $s=1$. The second solution exists only in three dimensions for any $s_{1}=s_{2}=s$. It has three derivatives for any $s$

$$
\mathcal{V}_{s, s, 1}=y_{1} y_{2} y_{3} z_{3}^{s-1}
$$

In higher dimensions, the number of derivatives rises with $s$ for $(s, s, 1)$ couplings, and a three-derivative vertex exists only for $s=1,2$. Instead, in $D=3$, the Maxwell field addresses all the spins $s \geq 2$ in an equal manner, but distinguishes them from spins $s=0,1$, that allow for coupling with one derivative. Only for $s=1$ are there both vertices-one-derivative and three-derivative. This is the only example in three dimensions in which more than one vertex for a given triple exists.

Coupling to gravity.-We confirm the presence of minimal coupling to gravity for any spin $[20,21]$ at the expense of deforming the gauge transformation of the gravitational field itself. The corresponding vertex is given by

$$
\mathcal{V}_{s, s, 2}=y_{3} z_{3}^{s-1}\left(s y_{1} z_{1}+s y_{2} z_{2}+y_{3} z_{3}\right),
$$

which is gauge invariant due to identities (10b). Remarkably, this expression makes sense for any $s$, including $s=0$. In the case of $s=2$, one has to use identities (10a) to show that (15) is equivalent to the conventional massless spin two self-interaction (EinsteinHilbert) vertex in any dimensions $\mathcal{V}_{2,2,2}=G^{2}$.

General triples.-Our analysis suggests (see Sec. IV of Ref. [24]) that all the vertices with $s_{1} \geq s_{2} \geq 2$ have common features. There is a unique vertex if the three spins satisfy strict triangle inequalities: $s_{i+1}+s_{i-1}>s_{i}$. We find no cubic vertices for triples, violating these inequalities. All of the cubic vertices with $s_{1} \geq s_{2} \geq 2$ fall into two classes- two derivative vertices (for an even sum of spins) and three derivative vertices (for an odd sum).

The case of two-derivative vertices corresponds to the even sum of spins. It is straightforward to show using the identities (10a) that the basis of independent monomials, second order in $y_{i} z_{i}$ is given by three monomials $y_{i+1} z_{i+1} y_{i-1} z_{i-1} \sim y_{i} z_{i} G$. Therefore, the most general ansatz for the two-derivative TT vertex in this case is

$$
\mathcal{V}=\left(\alpha_{1} y_{1} z_{1}+\alpha_{2} y_{2} z_{2}+\alpha_{3} y_{3} z_{3}\right) G z_{1}^{n_{1}} z_{2}^{n_{2}} z_{3}^{n_{3}}
$$

Gauge variations of this vertex will be three-derivative expressions. It is not hard to see, that using the identities (10a) and (10b) one can bring any monomial of these variations into a form, containing all three $y$ 's

$$
\begin{aligned}
\delta_{i} \mathcal{V}= & {\left[\alpha_{i}\left(n_{i+1}-n_{i-1}\right)+\left(\alpha_{i+1}-\alpha_{i-1}\right)\left(n_{i+1}+n_{i-1}+1\right)\right] } \\
& \times y_{1} y_{2} y_{3} z_{i} z_{1}^{n_{1}} z_{2}^{n_{2}} z_{3}^{n_{3}} .
\end{aligned}
$$

Gauge invariance conditions with respect to all three variations admit a unique solution, up to the overall constant

$$
\alpha_{i}=n_{i-1}+n_{i+1}+1=s_{i}-1
$$

therefore, the vertex can be written as

$\mathcal{V}=\left[\left(s_{1}-1\right) y_{1} z_{1}+\left(s_{2}-1\right) y_{2} z_{2}+\left(s_{3}-1\right) y_{3} z_{3}\right] G z_{1}^{n_{1}} z_{2}^{n_{2}} z_{3}^{n_{3}}$,

$n_{i}=\frac{1}{2}\left(s_{i-1}+s_{i+1}-s_{i}\right)-1 \geq 0$.

This vertex reproduces the minimal coupling to spin two (15) as a particular case. It can be made manifest, rewriting the vertex (19) in an equivalent form

$$
\begin{aligned}
\mathcal{V}_{s_{1}, s_{2}, s_{3}}= & y_{3} z_{1}^{n_{1}} z_{2}^{n_{2}} z_{3}^{n_{3}+1}\left[\left(s_{2}+s_{3}-2\right) y_{1} z_{1}\right. \\
& \left.+\left(s_{3}+s_{1}-2\right) y_{2} z_{2}+\left(s_{3}-1\right) y_{3} z_{3}\right] .
\end{aligned}
$$

For $s_{3}=2, s_{1}=s_{2}=s$, this reproduces the minimal coupling to gravity, given in (15). Now, we understood that the minimal coupling to gravity is a part of a bigger family of two-derivative vertices in three dimensions, that exist for every triple of integer spins greater than one, with an even sum and satisfying strict triangle inequalities.

The case of three-derivative vertices corresponds to the odd sum of spins. As long as the triangle inequalities between the spins are satisfied, any three-derivative vertex monomial contains third order polynomials in $y_{i} z_{i}$ 's and can be uniquely written in the form containing all the $y_{i}$ 's (we omit the arbitrary coupling constant in front)

$$
\begin{aligned}
& \mathcal{V}_{s_{1}, s_{2}, s_{3}}=y_{1} y_{2} y_{3} z_{1}^{n_{1}} z_{2}^{n_{2}} z_{3}^{n_{3}}, \\
& n_{i-1}+n_{i+1}+1=s_{i} .
\end{aligned}
$$

This expression is gauge invariant due to identities (10c). This vertex exists for any spins with the odd sum $s_{1}+s_{2}+s_{3}$ satisfying strict triangle inequalities. Three-derivative vertices $(s, s, 1)$ and $(s+1, s, 2)$ are of this type.

Because of the four-derivative Schouten identities (10c), any nontrivial vertex term with $n \geq 4$ derivatives contains at least $n-1$ powers of one of the $y_{i}$ 's. One can carefully consider all options and show that all the gauge invariant cubic vertices with more than three derivatives are those with scalar and Maxwell fields. We conclude, that there are no nontrivial interactions of fields with spins $s_{1} \geq s_{2} \geq s_{3} \geq 2$ 
with more than three derivatives. Since we already studied scalar and Maxwell cases in detail, this completes the classification of parity-even vertices of massless fields in three dimensions.

Discussion.-We have classified all parity-even cubic interactions between massless bosonic fields in three dimensions. A remarkable difference of three dimensional vertices compared to higher dimensional ones is that for any three spins $\left(s_{1}, s_{2}, s_{3}\right)$, there is at most a unique vertex (with the only exception of $s_{1}=s_{2}=s_{3}=1$ ). We refer to massless fields of spin 0 and 1, scalar and Maxwell fields, as "matter fields" since they carry propagating degrees of freedom in three dimensions. The vertices that coincide with the higher dimensional ones are all those containing at least two matter fields, spin two self-interaction (cubic vertex of Einstein-Hilbert action, see, e.g., [34]) and spin three couplings $(3, s, s)$ with $s \leq 3$. One more curiosity of this classification is that spin three couples to all spins through $(s, s, 3)$ couplings "universally" in three dimensions, similar to the spin two case (the latter property is associated to the equivalence principle)-all of these vertices have three derivatives.

The spin values, for which the vertex is absent in $D=3$, are those violating strict triangle inequality and containing one matter field at most. The vertices with $s_{1} \geq s_{2} \geq 2$ have two (three) derivatives for an even (odd) sum of spins in the vertex, and are non-Abelian. The only triple of spins that allows for more than one cubic vertex is $s_{1}=s_{2}=s_{3}=1$. In this case, there are two vertices-the Yang-Mills (YM) one, $\mathcal{V}_{\mathrm{YM}}=G$, and the $F^{3}$ one, $\mathcal{V}=y_{1} y_{2} y_{3}$, both requiring a fully antisymmetric color factor.

All cubic vertices in flat space can be uplifted to (A)dS space, therefore, via the AdS/CFT dictionary, the classification of cubic vertices in flat space should conform to the structure of three-point functions in 2D CFTs (see, e.g., [19]). Not only can the vertices described here be lifted to (A) $\mathrm{dS}_{3}$, but they can also be written in arbitrary Einstein background, covariantizing derivatives and treating gravity in a full nonlinear manner at the expense of deforming the gauge transformation of the metric itself, enlarging the isometry algebra to involve HS killing tensors (see, e.g., [35]). This is straightforward only in three dimensions and cubic vertices, due to the triviality of the Weyl tensor $[1,20,21]$. Although Einstein equations themselves get deformed due to couplings to HS fields, Einstein spaces are still classical solutions of the full theory with vanishing HS fields. The back reaction to Einstein equations should be taken into account when constructing quartic and higher order vertices, while it is not relevant at cubic order in HS fields.

The classification provided in this work is completely model independent. In order to compare to 2D CFT threepoint functions, we notice that the HS Fronsdal field with spin $s$ corresponds to a conserved current on the boundary which has two nonzero components-chiral and antichiral quasiprimaries with a conformal weight of $s$. The three-point functions of these currents are uniquely fixed by their conformal weights (see, e.g., [36]) and result in a unique parity-even three-point function for given triplet of spins when the chiral and antichiral pieces transform into each other under parity transformations; therefore, they allow for only one free parameter-a coincident overall constant. This is in agreement with our findings. The absence of the cubic vertices for certain spin values is in agreement with the structure of the $3 \mathrm{D}$ HS algebra $h s[\lambda]$, that has vanishing structure constants for three spins violating triangle inequalities [35]. As opposed to higher dimensions, there are no Abelian vertices in three dimensions for $s_{1} \geq s_{2} \geq 2$. To our best knowledge, it has not been observed in the 2D CFT literature that the three-point correlators of quasiprimaries with spins violating triangle inequalities must be zero. Therefore, we have uncovered a novel model-independent feature of 2D CFT's, which is, of course, consistent with known models with $W$-algebra symmetries [37]. Further discussion can be found in Supplemental Material [24] and the sequel paper [38].

An immediate application of the results presented here is the construction of the metriclike action up to cubic order for the Prokushkin-Vasiliev theory [2] with a symmetry of $h s[\lambda]$ for arbitrary $\lambda$. In the aforementioned theory, this free parameter manifests itself as the mass for the scalar field that appears as a vacuum expectation value of an auxiliary field. The mass of the scalar does not introduce complications compared to the massless case (see, e.g., $[39,40]$ ). We hope to report on this program in the near future.

The author is grateful to Pan Kessel for stimulating discussions that motivated this research, to Eduardo Conde, Dario Francia, Euihun Joung, Gabriele Lo Monaco, Rubik Poghossian, Stefan Theisen, and Arkady Tseytlin for useful discussions on the subject of this work, to Andrea Campoleoni and Stefan Fredenhagen for communication and to Jirayr Avetisyan, Karen Hovhannisyan, and Eugene Skvortsov for comments on the draft and useful suggestions. This work is supported by the Alexander von Humboldt Foundation.

*karapet.mkrtchyan@aei.mpg.de

[1] A. Campoleoni, S. Fredenhagen, S. Pfenninger, and S. Theisen, Towards metric-like higher-spin gauge theories in three dimensions, J. Phys. A 46, 214017 (2013); A. Campoleoni, S. Fredenhagen, and J. Raeymaekers, Quantizing higher-spin gravity in free-field variables, J. High Energy Phys. 02 (2018) 126.

[2] S. F. Prokushkin and M. A. Vasiliev, Higher spin gauge interactions for massive matter fields in 3-D AdS spacetime, Nucl. Phys. B545, 385 (1999); Cohomology of arbitrary spin currents in $\operatorname{AdS}(3)$, Teor. Mat. Fiz. 123, 3 (2000) [Theor. Math. Phys. 123, 415 (2000)]. 
[3] M. P. Blencowe, A consistent interacting massless higher spin field theory in $D=(2+1)$, Classical Quantum Gravity 6, 443 (1989).

[4] A. Campoleoni, S. Fredenhagen, S. Pfenninger, and S. Theisen, Asymptotic symmetries of three-dimensional gravity coupled to higher-spin fields, J. High Energy Phys. 11 (2010) 007.

[5] H. Afshar, A. Bagchi, R. Fareghbal, D. Grumiller, and J. Rosseel, Spin-3 Gravity in Three-Dimensional Flat Space, Phys. Rev. Lett. 111, 121603 (2013).

[6] S. Gwak, E. Joung, K. Mkrtchyan, and S. J. Rey, Rainbow valley of colored (anti) de Sitter gravity in three dimensions, J. High Energy Phys. 04 (2016) 055; Rainbow vacua of colored higher-spin (A) $\mathrm{dS}_{3}$ gravity, J. High Energy Phys. 05 (2016) 150; L. Eberhardt, M. R. Gaberdiel, and I. Rienacker, Higher spin algebras and large $\mathcal{N}=4$ holography, J. High Energy Phys. 03 (2018) 097.

[7] M. Banados, C. Teitelboim, and J. Zanelli, The Black Hole in Three-Dimensional Space-Time, Phys. Rev. Lett. 69, 1849 (1992).

[8] M. Henneaux and S. J. Rey, Nonlinear $W_{\text {infinity }}$ as asymptotic symmetry of three-dimensional higher spin antide Sitter gravity, J. High Energy Phys. 12 (2010) 007.

[9] M. R. Gaberdiel and R. Gopakumar, $\mathrm{An} \mathrm{AdS}_{3}$ dual for minimal model CFTs, Phys. Rev. D 83, 066007 (2011); M. R. Gaberdiel and R. Gopakumar, Minimal model holography, J. Phys. A 46, 214002 (2013).

[10] A. Achucarro and P. K. Townsend, A Chern-Simons action for three-dimensional anti-de Sitter supergravity theories, Phys. Lett. B 180, 89 (1986).

[11] S. Fredenhagen and P. Kessel, Metric- and frame-like higher-spin gauge theories in three dimensions, J. Phys. A 48, 035402 (2015).

[12] A. Campoleoni and M. Henneaux, Asymptotic symmetries of three-dimensional higher-spin gravity: The metric approach, J. High Energy Phys. 03 (2015) 143.

[13] C. Fronsdal, Massless fields with integer spin, Phys. Rev. D 18, 3624 (1978).

[14] R. Manvelyan, K. Mkrtchyan, and W. Rühl, General trilinear interaction for arbitrary even higher spin gauge fields, Nucl. Phys. B836, 204 (2010).

[15] E. Conde, E. Joung, and K. Mkrtchyan, Spinor-helicity three-point amplitudes from local cubic interactions, J. High Energy Phys. 08 (2016) 040.

[16] A. Sagnotti and M. Taronna, String lessons for higher-spin interactions, Nucl. Phys. B842, 299 (2011).

[17] A. Fotopoulos and M. Tsulaia, On the tensionless limit of string theory, off-shell higher spin interaction vertices and BCFW recursion relations, J. High Energy Phys. 11 (2010) 086.

[18] R. Manvelyan, K. Mkrtchyan, and W. Rühl, A Generating function for the cubic interactions of higher spin fields, Phys. Lett. B 696, 410 (2011).

[19] M. S. Costa, J. Penedones, D. Poland, and S. Rychkov, Spinning conformal correlators, J. High Energy Phys. 11 (2011) 071.

[20] C. Aragone and S. Deser, Hypersymmetry in $D=3$ of coupled gravity massless spin 5/2 system, Classical Quantum Gravity 1, L9 (1984).
[21] Y. M. Zinoviev, Hypergravity in $\mathrm{AdS}_{3}$, Phys. Lett. B 739 , 106 (2014).

[22] A. K. H. Bengtsson, I. Bengtsson, and N. Linden, Interacting higher spin gauge fields on the light front, Classical Quantum Gravity 4, 1333 (1987).

[23] R. R. Metsaev, Cubic interaction vertices of massive and massless higher spin fields, Nucl. Phys. B759, 147 (2006).

[24] See Supplemental Material at http://link.aps.org/ supplemental/10.1103/PhysRevLett.120.221601 for details of derivations and expanded discussion, which includes Refs. [25-33].

[25] A. Campoleoni and D. Francia, Maxwell-like Lagrangians for higher spins, J. High Energy Phys. 03 (2013) 168.

[26] E. Joung, L. Lopez, and M. Taronna, Solving the Noether procedure for cubic interactions of higher spins in (A)dS, J. Phys. A 46, 214020 (2013).

[27] R. Manvelyan, K. Mkrtchyan, and W. Ruhl, Off-shell construction of some trilinear higher spin gauge field interactions, Nucl. Phys. B826, 1 (2010).

[28] K. Mkrtchyan, Higher spin interacting quantum field theory and higher order conformal invariant Lagrangians, $\mathrm{PhD}$ thesis, Department of Theoretical Physics, Yerevan Physics Institute, 2010, arXiv:1011.0160.

[29] R. Manvelyan and K. Mkrtchyan, Conformal invariant interaction of a scalar field with the higher spin field in AdS(D), Mod. Phys. Lett. A 25, 1333 (2010).

[30] X. Bekaert, E. Joung, and J. Mourad, On higher spin interactions with matter, J. High Energy Phys. 05 (2009) 126.

[31] E. Joung and M. Taronna, Cubic interactions of massless higher spins in (A)dS: metric-like approach, Nucl. Phys. B861, 145 (2012).

[32] C. Aragone and S. Deser, Consistency problems of hypergravity, Phys. Lett. 86B, 161 (1979).

[33] F. A. Berends, G. J. H. Burgers, and H. van Dam, On the theoretical problems in constructing interactions involving higher spin massless particles, Nucl. Phys. B260, 295 (1985).

[34] R. Manvelyan, K. Mkrtchyan, and W. Rühl, Direct construction of a cubic selfinteraction for higher spin gauge fields, Nucl. Phys. B844, 348 (2011).

[35] E. Joung and K. Mkrtchyan, Notes on higher-spin algebras: minimal representations and structure constants, J. High Energy Phys. 05 (2014) 103.

[36] R. Blumenhagen and E. Plauschinn, Introduction to conformal field theory: With applications to String theory, Lect. Notes Phys. 779, 1 (2009).

[37] A. Campoleoni, S. Fredenhagen, and S. Pfenninger, Asymptotic $\mathrm{W}$-symmetries in three-dimensional higher-spin gauge theories, J. High Energy Phys. 09 (2011) 113.

[38] P. Kessel and K. Mkrtchyan, Cubic interactions of massless bosonic fields in three dimensions II: Parity-odd and Chern-Simons vertices, Phys. Rev. D 97, 106021 (2018).

[39] M. Ammon, P. Kraus, and E. Perlmutter, Scalar fields and three-point functions in D $=3$ higher spin gravity, J. High Energy Phys. 07 (2012) 113.

[40] I. Lovrekovic, Three dimensional higher spin holography, arXiv:1801.00772. 